\title{
Erythrocyte Morphological Characteristics Based on Microscope Images System
}

\author{
Bohua Feng, Liufen Peng* \\ College of Medical Information Engineering, Guangdong Pharmaceutical University, Guangzhou, P. R. China
}

Email address:

tfengbh@163.com (Liufen Peng)

${ }^{*}$ Corresponding author

To cite this article:

Bohua Feng, Liufen Peng. Erythrocyte Morphological Characteristics Based on Microscope Images System. International Journal of Biomedical Science and Engineering. Vol. 4, No. 3, 2016, pp. 22-27. doi: 10.11648/j.ijbse.20160403.12

Received: August 30, 2016; Accepted: September 9, 2016; Published: September 27, 2016

\begin{abstract}
Microscope images analysis of erythrocyte (red blood cells, RBCs) was a widely used method for medical purpose. Usually manual measuring and analysis of the images were subject to time-consuming, errors and unstability of results. The images analysis method had been combined with computer image processing techniques in this research. A measuring and analysis system for microscope images(MIAS) of RBCs was developed, which could recognize RBCs in images and measure cells mophometric parameters. Normal human RBCs were compared with ones under high glucose. The results indicated RBCs sizes parameters such as areas, perimeters, major axis lengths, minor axis lengths, elongations, roundnesses and Feret diameters have difference between normal and high glucose conditions. RBCs normal disk shapes changed into acanthocyte and stomatocyte under higher glucose conditions. This fast and precise method for measuring RBCs morphometric parameters contributed to pathogenesis of diabetic nephropathy(DN) research.
\end{abstract}

Keywords: Diabetic Nephropathy, Microscope Image Analysis, Morphological Characteristics

\section{Introduction}

The pathogenesis of diabetic nephropathy (DN) was complex, and the present study on DN is still not clear and systematic. Blood rheology examination showed that the DN patients blood glucose levels are high, the red blood cells (RBCs) are in hypertonic condition, which enhance the cell membrane protein glycosylation. Then the RBCs deformability, oxygen release amount, enzyme activity were definitely decreased. [1-3] These changes directly affected tissue blood supply, resulting in renal tissue damage [4]. Structure alterations in RBCs of DN patients of RBCs had important influence on their function and protein expression, RBCs shape and structure parameters were contribute to clearly understanding the pathogenesis, detection and control method of DN [5]. Computer image processing and pattern recognition technology of microscopic image of RBCs images had important significance in medical research and clinical application. Compared to artificial way, computer image processing and analysis improved the work efficiency, and enhanced the objectivity of the analysis [6]. In this study,
RBCs samples were prepared, then RBC microscopic image analysis system (MIAS) were developed. The MIAS achieved a variety methods of image processing, including image enhancement, image point operations, color filling, edge detection. The image analysis module mainly realized the measurement of RBCs shape parameters, including searching center, RBCs counting, size and shape parameters calculating. Through a comparative study on the measuring results, qualitative and quantitative characteristics of $\mathrm{RBC}$ morphological parameters were implemented especially abnormal RBCs under high glucose condition were researched.

\section{Methods}

\subsection{Prepartion of Phosphate Buffered Saline (PBS)}

The PBS was prepared as follows: $2.5 \mathrm{~g} \mathrm{NaH}_{2} \mathrm{PO}_{4} \cdot \mathrm{H}_{2} \mathrm{O}$, $22.49 \mathrm{~g} \mathrm{Na}_{2} \mathrm{HPO}_{4} \cdot 7 \mathrm{H}_{2} \mathrm{O}$ and $175.33 \mathrm{~g} \mathrm{NaCl}$ were dissolved in distilled water. Then the solution was moved into $1000 \mathrm{~mL}$ 
volumetric flask, adding distilled water to its full scale. The PBS solution was then placed in the refrigerator under $4^{\circ} \mathrm{C}$. The $95 \mathrm{~mL}$ PBS was then blent with glucose $0.090 \mathrm{~g}$. The mixing solution's $\mathrm{pH}$ was adjusted to 7.4. The PBS solution containing $5 \mathrm{mM}$ phosphate, $150 \mathrm{NaCl} \mathrm{mM}$ and $5 \mathrm{mM}$ glucose. Before using the PBS, appropriate amount of bovine serum albumin would be added to maintain RBCs shape. [7]

\subsection{Device}

Nikon TE300 fluorescence phase contrast inverted microscope $(40 \times, \mathrm{ph} 2)$, high sensitivity $\mathrm{CCD}$ camera (Sumsung, Korea), thermostatic waterbath, centrifuge, the sodium citrate anticoagulation $(0.109 \mathrm{~mol} / \mathrm{L}$, Sodium Citrate 1:9, Guangzhou Yang Pu medical Co., Ltd.), transferpettors (Dragon lab, Beijing), computer (Lenovo, Pentium Dual-Core E5300 2.6GHz, China), Micrometer for microscope (scale division $0.01 \mathrm{~mm}$ ).

\subsection{Preparation of $\mathrm{RBCs}$}

The venous bloods were collected from healthy adult (Group A, male, 34 years old), and DN patient (Group B, male, 58 years old, moderate and severe renal dysfunction with glomerular filtration rate (GFR) $\left.15-60 \mathrm{ml} / \mathrm{min} \cdot 1.73 \mathrm{~m}^{2}\right)[8,9]$. The samples anticoagulated with sodium hepari were removed white blood cells and platelets in upper layer after centrifugation. RBCs were then mixed with PBS. The volume ratio of PBS and RBCs was 10 to 1 . After centrifugalization and washing, young RBCs of Group A were obtained and suspended in PBS with glucose solution by the concentration of $20 \mathrm{mmol} / \mathrm{L}$ and $5 \mathrm{mmol} / \mathrm{L}$.

\subsection{RBCs Microscopic Image Processing and Characterization}

After the micrometer calibration, the RBCs slides were prepared and put on microscope stage in the constant temperature sample pool. The field diaphragm, cursory and accurate focusing were adjusted until a bright clear image was observed under $40 \times$ objective lens. Then the charge coupled device (CCD) was used to aquire the RBC images which would be converted into digital signal by image acquisition card. The digital signal was saved as computer image format file. Using the MIAS to measure the RBCs geometric parameters such as the contact area, perimeter, major axis length, minor axis length, elongation, roundness and Feret diameter, 15-20 RBCs were investigated at each glucose gradient concentration where repeated 3 times.

\section{Results and Discussion}

\subsection{Morphology and Structure of RBCs}

The normal human RBC morphology is smooth edge discocyte of diameter 7-9 $\mu \mathrm{m}$, as shown in figure 1a and figure $2 \mathrm{a}$.
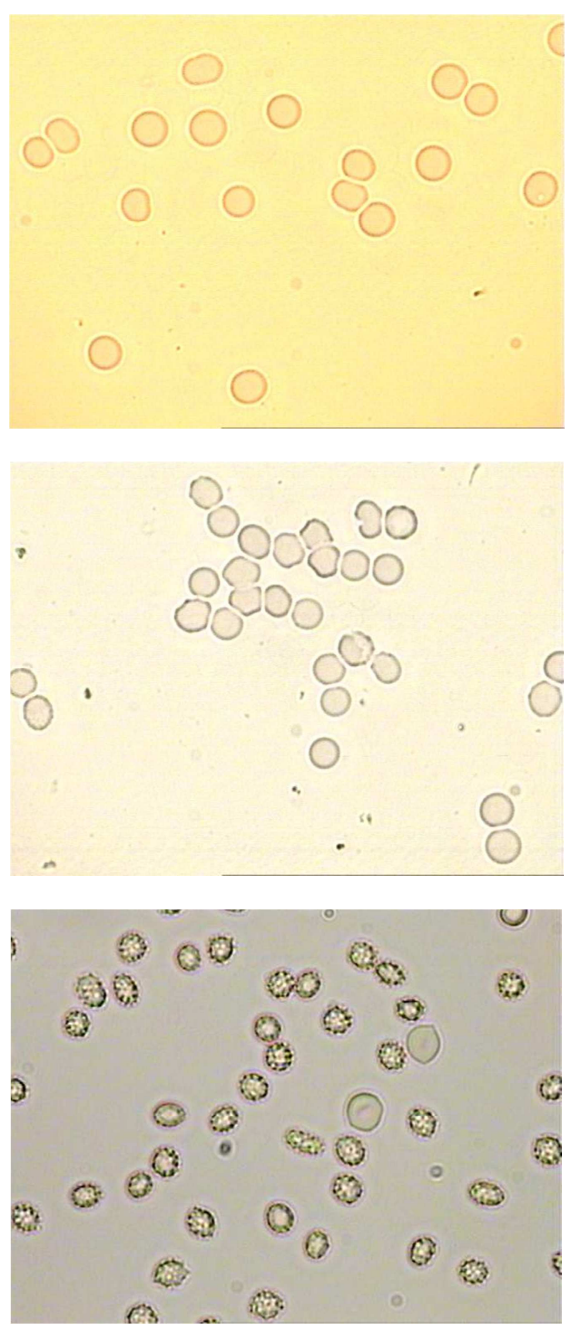

Figure 1. Normal RBCs (a), RBCs under high glucose concentration (b), DN $\operatorname{RBCs}(c)$.
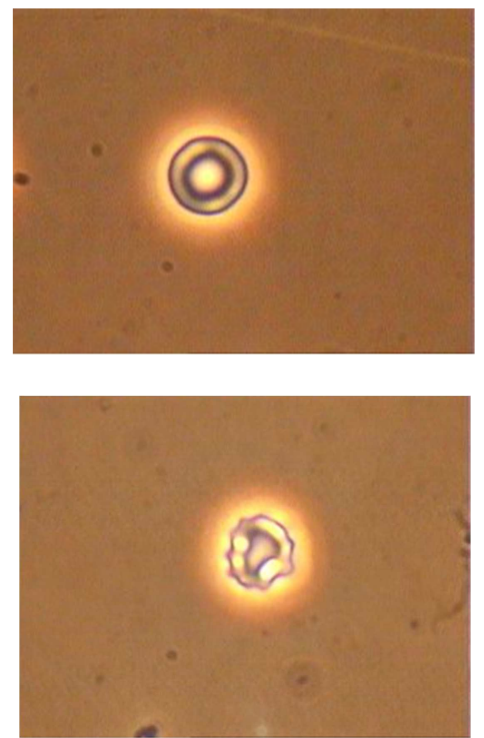

Figure 2. A single living $R B C$ in normal shape (a), under high glucose concentration (b).

Under a certain glucose concentration, the RBCs shapes changed obviously. Though RBCs sizes were similar in figure 
$1 \mathrm{a}$ and $\mathrm{b}$, the cells edges in figure $1 \mathrm{~b}$ became irregular. Many RBCs were adhered to each other, some cells even showed stomatocyte shape, as shown in figure $1 \mathrm{~b}$. Further increasing the glucose concentration, a considerable number of RBCs sizes reduced and changed into the shape of acanthocyte [10] with lots of vesicles on cell membrane, as shown in figure $1 \mathrm{c}$. The normal single living RBC shown in figure 2a was obviously more smooth and the acanthocyte one as shown in figure $2 \mathrm{~b}$. Therefore glucose concentration had a significant effect on RBCs shape.

\subsection{Microscope Images Enhancement and Binarization}

The RBCs images quality were usually low contrast and high noise, which had effect on image segmentation processing. Therefore, median filtering technique was applied to removing image noise. A pixel with its coordinates $(\mathrm{x}, \mathrm{y})$ was set as a center point in the microscope image, around which pixels distributed within the range of $3 \times 3$ area were sorted in ascending order of gray levels. And the mid-value of those pixels gray levels was considered as the gray level of the center pixel. This procedure was achieved by transferring original image pixels data to buffer, then affirmative mid-value of each pixel in buffer was copied to image to overwrite the original data. Thus, the RBCs in microscope images had more smooth edges and lower noise distinguished from background, which was contributed to further analysis. Image point operation was implemented by histogram thresholding method. The distribution of gray levels in microscope images were shown in figure 3 as a 2-dimension histogram, where horizontal ordinate referred to gray levels, and vertical coordinate represented gray levels frequency in RBCs images. The scope of gray levels threshold was easily adjusted in MIAS to achieve image binarization. And gray levels of points within the threshold range were marked by MIAS automaticly. The closed regions referring to RBCs were filled by specified color as shown in figure 3 .

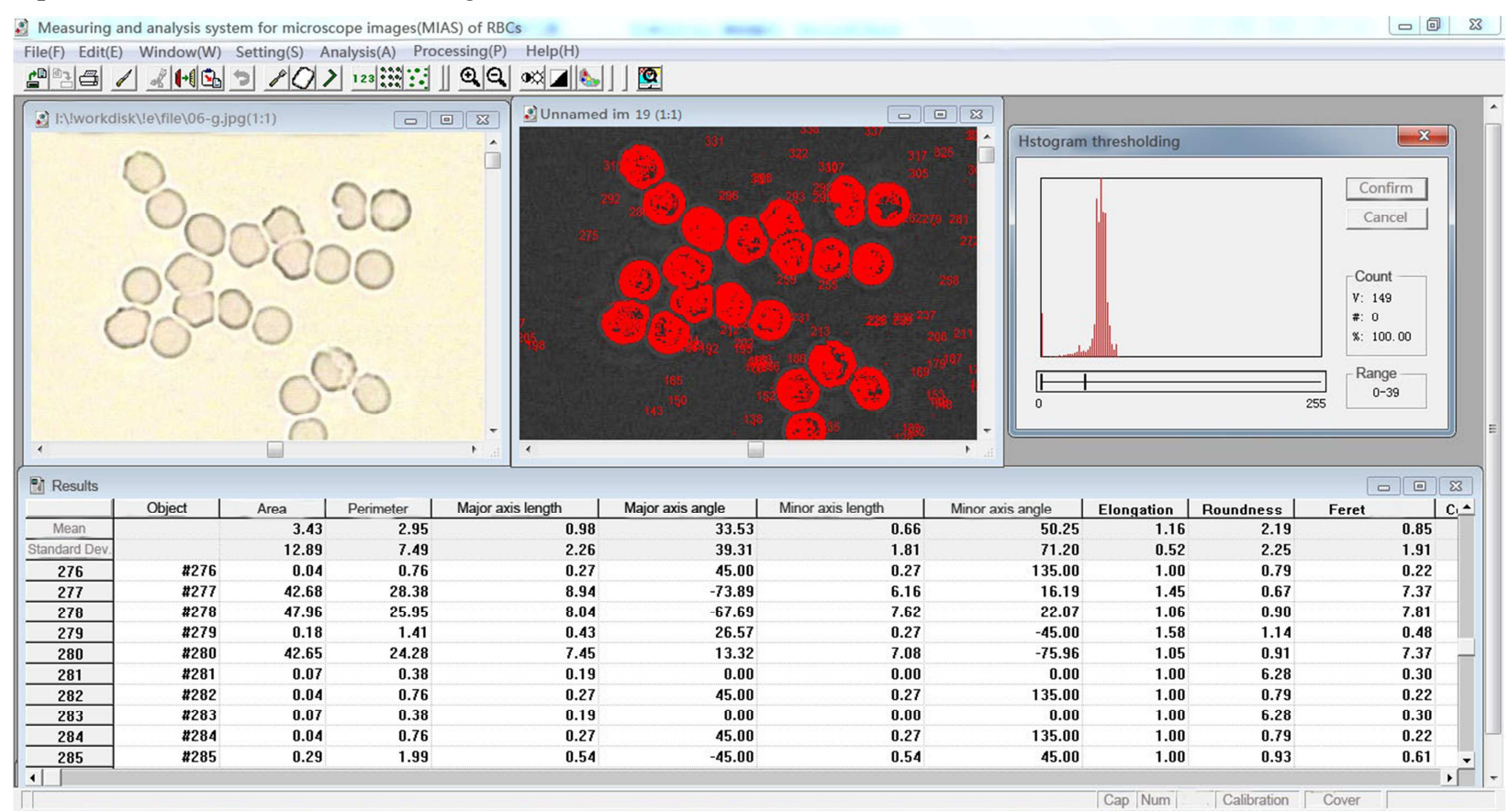

Figure 3. Adjusting histogram thresholding and binarization.

\subsection{RBCs Edges Detection Algorithm}

RBCs edges in microscope image were investigated by means of Sobel operator, which could decrease noise and obtain accurate edges. [11] Adjacent pixels gray level weighted difference reached the maximum value at $\mathrm{RBCs}$ edges. The operator contained two $3 \times 3$ convolution kernels, whose row and column number is odd. One was for detecting level edges, the other was for the vertical edges. After plane convolution with the operator, the image's horizontal and vertical brightness difference approximations were obtained. The calculation process was shown in scheme (1) to (3), where A represented original image, Gx and Gy separately standed for image edge detected in horizontal and vertical directions. Gray gradient value of single pixel were thus calculated in the two directions by scheme (2). Furthermore, gray gradient direction angle $\theta$ in scheme (3) was gained, where $\theta$ equaled to zero indicating a longitudinal edge of $\mathrm{RBC}$ with higher brightness in the right side of its edge.

$$
\begin{aligned}
\mathrm{G}_{\mathrm{x}}=\left[\begin{array}{lll}
-1 & 0 & +1 \\
-2 & 0 & +2 \\
-1 & 0 & +1
\end{array}\right] * \text { A } & \text { and } \mathrm{G}_{\mathrm{y}}=\left[\begin{array}{ccc}
+1 & +2 & +1 \\
0 & 0 & 0 \\
-1 & -2 & -1
\end{array}\right] * A \\
\mathrm{G} & =\sqrt{\mathrm{G}_{\mathrm{x}}^{2}+\mathrm{G}_{\mathrm{y}}^{2}} \\
\theta & =\arctan \left(\frac{\mathrm{G}_{\mathrm{y}}}{\mathrm{G}_{\mathrm{x}}}\right)
\end{aligned}
$$

Some RBCs edges were blurred in the microscope image. As Sobel operator method was implemented, noise was eliminated and pixels of large gradient values were removed. 
Additionally, many small meaningless points should not be considered as RBCs. Corrosion and expansion functions were applied to remove these points in binarized image (figure 4). All those processes above prepared a clear image compared to the original one, and the RBCs were specifically recognized eliminating the noise. As shown in figure 4, the RBCs were correctly selected by drawing a red line along their perimeters.

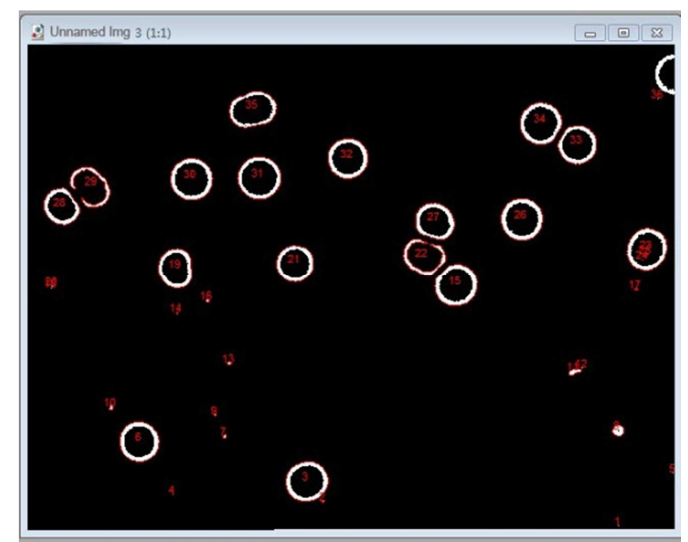

Figure 4. RBCs edges detection and marking.

\subsection{RBC Shape Data Acquisition and Analysis}

Image analysis module aimed to search RBCs center point and calculate shape parameters such as area, perimeter, major axis length, minor axis length, elongation, roundness and Feret diameter. After many times corrosions and thinning, image of obvious RBCs had been shown in figure 3, where the red color marked shapes made the center point searching and parameters calculation easier. The centers of each RBC were counted automatically, shape parameters were calculated using geometric formulas by MIAS. Though pixel was considered as a suitable arithmatical unit in MIAS, international units(IU) were more useful for researchers respectively. So MIAS showed the calculation results in IU form.

When RBCs were in normal glucose level solution $(\mathrm{pH}=7.4)$, their shapes parameters were calculated and shown by MIAS in table1. And under the high concentration of glucose, a stomatocyte shape cells parameters were listed in table 2 .

Table 1. Normal RBCs shape parameters (unit: $\mu \mathrm{m})$.

\begin{tabular}{|c|c|c|c|c|c|c|c|}
\hline & Area $\left[\mu m^{2}\right]$ & Perimeter & $\begin{array}{l}\text { Major Axis } \\
\text { Length }\end{array}$ & $\begin{array}{l}\text { Minor Axis } \\
\text { Length }\end{array}$ & Elongation & Roundness & $\begin{array}{l}\text { Feret } \\
\text { Diameter }\end{array}$ \\
\hline 1 & $48.84 \pm 3.09$ & $25.60 \pm 5.01$ & $8.14 \pm 0.30$ & $7.36 \pm 0.12$ & $1.11 \pm 0.06$ & $0.94 \pm 0.03$ & $7.89 \pm 0.08$ \\
\hline 2 & $45.40 \pm 4.71$ & $24.93 \pm 7.59$ & $7.65 \pm 0.15$ & $7.38 \pm 0.46$ & $1.04 \pm 0.06$ & $0.92 \pm 0$ & $7.60 \pm 0.23$ \\
\hline 3 & $50.74 \pm 6.00$ & $26.47 \pm 5.38$ & $8.30 \pm 0.07$ & $7.50 \pm 0.12$ & $1.11 \pm 0.07$ & $0.91 \pm 0.01$ & $8.04 \pm 0.40$ \\
\hline 4 & $39.62 \pm 6.84$ & $23.47 \pm 6.27$ & $7.63 \pm 0.07$ & $6.60 \pm 0.32$ & $1.16 \pm 0.05$ & $0.9 \pm 0.02$ & $7.10 \pm 0.20$ \\
\hline 5 & $39.59 \pm 6.57$ & $23.10 \pm 5.05$ & $7.19 \pm 0.11$ & $6.93 \pm 0.04$ & $1.04 \pm 0.06$ & $0.93 \pm 0.02$ & $7.10 \pm 0.05$ \\
\hline 6 & $14.15 \pm 6.16$ & $44.40 \pm 7.74$ & $8.17 \pm 0.50$ & $5.99 \pm 0.48$ & $1.36 \pm 0.05$ & $0.09 \pm 0.01$ & $4.24 \pm 0.21$ \\
\hline 7 & $47.92 \pm 3.48$ & $27.06 \pm 7.18$ & $8.18 \pm 0.24$ & $7.38 \pm 0.10$ & $1.11 \pm 0.01$ & $0.82 \pm 0.02$ & $7.81 \pm 0.42$ \\
\hline 8 & $50.70 \pm 5.11$ & $26.09 \pm 6.44$ & $8.12 \pm 0.36$ & $7.87 \pm 0.05$ & $1.03 \pm 0.08$ & $0.94 \pm 0.01$ & $8.03 \pm 0.04$ \\
\hline 9 & $40.76 \pm 6.10$ & $23.85 \pm 7.24$ & $7.65 \pm 0.10$ & $6.69 \pm 0.29$ & $1.14 \pm 0.10$ & $0.90 \pm 0.01$ & $7.20 \pm 0.42$ \\
\hline 10 & $35.57 \pm 6.72$ & $22.60 \pm 7.18$ & $7.12 \pm 0.47$ & $5.84 \pm 0.15$ & $1.22 \pm 0.04$ & $0.87 \pm 0.01$ & $6.73 \pm 0.03$ \\
\hline 11 & $12.83 \pm 6.88$ & $43.35 \pm 5.91$ & $8.40 \pm 0.05$ & $6.10 \pm 0.42$ & $1.38 \pm 0.05$ & $0.09 \pm 0.02$ & $4.04 \pm 0.09$ \\
\hline 12 & $50.74 \pm 6.54$ & $26.20 \pm 7.02$ & $8.07 \pm 0.42$ & $7.80 \pm 0.34$ & $1.03 \pm 0.02$ & $0.93 \pm 0.02$ & $8.04 \pm 0.25$ \\
\hline 13 & $52.64 \pm 6.01$ & $26.67 \pm 5.94$ & $8.25 \pm 0.38$ & $8.07 \pm 0.38$ & $1.02 \pm 0.01$ & $0.93 \pm 0.02$ & $8.19 \pm 0.26$ \\
\hline 14 & $46.24 \pm 4.68$ & $25.39 \pm 6.91$ & $7.71 \pm 0.17$ & $7.45 \pm 0.36$ & $1.03 \pm 0.06$ & $0.90 \pm 0.05$ & $7.67 \pm 0.14$ \\
\hline 15 & $42.15 \pm 5.81$ & $24.52 \pm 5.36$ & $7.39 \pm 0.26$ & $7.21 \pm 0.36$ & $1.02 \pm 0.04$ & $0.88 \pm 0.04$ & $7.33 \pm 0.31$ \\
\hline 16 & $49.31 \pm 3.42$ & $27.08 \pm 6.16$ & $8.03 \pm 0.48$ & $7.76 \pm 0.20$ & $1.03 \pm 0.08$ & $0.84 \pm 0.02$ & $7.92 \pm 0.45$ \\
\hline 17 & $48.22 \pm 6.15$ & $27.29 \pm 7.58$ & $9.14 \pm 0.50$ & $6.36 \pm 0.12$ & $1.44 \pm 0.01$ & $0.81 \pm 0.01$ & $7.84 \pm 0.20$ \\
\hline Mean & 42.08 & 27.53 & 7.95 & 7.08 & 1.13 & 0.8 & 7.22 \\
\hline Standard Deviation & 11.79 & 6.32 & 0.5 & 0.7 & 0.14 & 0.27 & 1.23 \\
\hline
\end{tabular}

Table 2. RBCs shape parameters under high glucose concentration (unit: $\mu m$ ).

\begin{tabular}{|c|c|c|c|c|c|c|c|}
\hline & Area $\left(\mu \mathrm{m}^{2}\right)$ & Perimeter & $\begin{array}{l}\text { Major Axis } \\
\text { Length }\end{array}$ & $\begin{array}{l}\text { Minor Axis } \\
\text { Length }\end{array}$ & Elongation & Roundness & Feret Diameter \\
\hline 1 & $51.54 \pm 4.44$ & $27.16 \pm 7.32$ & $8.24 \pm 0.01$ & $8.06 \pm 0.31$ & $1.02 \pm 0.07$ & $0.88 \pm 0.04$ & $8.10 \pm 0.41$ \\
\hline 2 & $49.68 \pm 3.04$ & $27.96 \pm 7.46$ & $8.76 \pm 0.06$ & $7.80 \pm 0.36$ & $1.12 \pm 0.06$ & $0.80 \pm 0.04$ & $7.95 \pm 0.30$ \\
\hline 3 & $47.96 \pm 3.11$ & $26.09 \pm 7.49$ & $7.92 \pm 0.48$ & $7.74 \pm 0.12$ & $1.02 \pm 0.05$ & $0.89 \pm 0.05$ & $7.81 \pm 0.36$ \\
\hline 4 & $47.23 \pm 4.24$ & $25.65 \pm 6.84$ & $7.99 \pm 0.34$ & $7.72 \pm 0.45$ & $1.03 \pm 0.09$ & $0.90 \pm 0.03$ & $7.75 \pm 0.39$ \\
\hline 5 & $46.94 \pm 6.70$ & $27.43 \pm 7.89$ & $8.14 \pm 0.02$ & $7.36 \pm 0.4$ & $1.11 \pm 0.04$ & $0.78 \pm 0.05$ & $7.73 \pm 0.50$ \\
\hline 6 & $45.29 \pm 4.95$ & $25.01 \pm 6.02$ & $8.34 \pm 0.03$ & $7.11 \pm 0.24$ & $1.17 \pm 0.03$ & $0.91 \pm 0.05$ & $7.59 \pm 0.11$ \\
\hline 7 & $43.79 \pm 7.51$ & $26.76 \pm 6.16$ & $8.14 \pm 0.46$ & $6.98 \pm 0.02$ & $1.17 \pm 0.04$ & $0.77 \pm 0.04$ & $7.47 \pm 0.33$ \\
\hline 8 & $42.26 \pm 5.33$ & $24.02 \pm 5.94$ & $7.92 \pm 0.09$ & $6.57 \pm 0.49$ & $1.21 \pm 0.01$ & $0.92 \pm 0.03$ & $7.34 \pm 0.16$ \\
\hline 9 & $42.11 \pm 3.95$ & $25.49 \pm 6.04$ & $7.81 \pm 0.06$ & $6.96 \pm 0.41$ & $1.12 \pm 0.02$ & $0.81 \pm 0.05$ & $7.32 \pm 0.20$ \\
\hline 10 & $41.31 \pm 6.87$ & $24.40 \pm 7.69$ & $7.39 \pm 0.01$ & $7.20 \pm 0.39$ & $1.03 \pm 0.02$ & $0.87 \pm 0.03$ & $7.25 \pm 0.32$ \\
\hline 11 & $41.20 \pm 7.92$ & $24.40 \pm 6.30$ & $7.47 \pm 0.16$ & $7.28 \pm 0.29$ & $1.03 \pm 0.08$ & $0.87 \pm 0.03$ & $7.24 \pm 0.07$ \\
\hline 12 & $41.01 \pm 7.92$ & $24.04 \pm 5.39$ & $7.32 \pm 0.09$ & $7.18 \pm 0.02$ & $1.02 \pm 0.07$ & $0.89 \pm 0.02$ & $7.23 \pm 0.19$ \\
\hline 13 & $40.72 \pm 5.42$ & $24.52 \pm 5.35$ & $7.74 \pm 0.37$ & $6.96 \pm 0.27$ & $1.11 \pm 0.05$ & $0.85 \pm 0.01$ & $7.20 \pm 0.12$ \\
\hline 14 & $39.84 \pm 3.11$ & $23.63 \pm 7.97$ & $7.23 \pm 0.15$ & $6.96 \pm 0.18$ & $1.04 \pm 0.05$ & $0.90 \pm 0.03$ & $7.12 \pm 0.24$ \\
\hline
\end{tabular}




\begin{tabular}{|c|c|c|c|c|c|c|c|}
\hline & Area $\left(\mu \mathrm{m}^{2}\right)$ & Perimeter & $\begin{array}{l}\text { Major Axis } \\
\text { Length }\end{array}$ & $\begin{array}{l}\text { Minor Axis } \\
\text { Length }\end{array}$ & Elongation & Roundness & Feret Diameter \\
\hline 15 & $39.48 \pm 7.72$ & $23.55 \pm 5.21$ & $7.18 \pm 0.18$ & $6.91 \pm 0.39$ & $1.04 \pm 0.01$ & $0.89 \pm 0.04$ & $7.09 \pm 0.43$ \\
\hline 16 & $38.53 \pm 5.09$ & $24.20 \pm 5.92$ & $7.27 \pm 0.12$ & $7.00 \pm 0.10$ & $1.04 \pm 0.06$ & $0.83 \pm 0.03$ & $7.00 \pm 0.01$ \\
\hline 17 & $37.91 \pm 3.57$ & $22.93 \pm 7.2$ & $7.21 \pm 0.18$ & $7.05 \pm 0.16$ & $1.02 \pm 0.04$ & $0.91 \pm 0.04$ & $6.95 \pm 0.31$ \\
\hline 18 & $37.29 \pm 3.49$ & $23.01 \pm 7.47$ & $7.08 \pm 0.14$ & $6.81 \pm 0.13$ & $1.04 \pm 0.01$ & $0.89 \pm 0.02$ & $6.89 \pm 0.19$ \\
\hline 19 & $36.96 \pm 4.74$ & $23.43 \pm 6.55$ & $7.58 \pm 0.46$ & $6.63 \pm 0.06$ & $1.14 \pm 0.01$ & $0.85 \pm 0.04$ & $6.86 \pm 0.25$ \\
\hline 20 & $33.52 \pm 7.80$ & $84.58 \pm 6.55$ & $13.55 \pm 0.15$ & $8.52 \pm 0.25$ & $1.59 \pm 0.08$ & $0.06 \pm 0.04$ & $6.53 \pm 0.49$ \\
\hline 21 & $24.38 \pm 7.74$ & $54.10 \pm 5.92$ & $8.23 \pm 0.13$ & $7.66 \pm 0.38$ & $1.07 \pm 0.04$ & $0.10 \pm 0.03$ & $5.57 \pm 0.13$ \\
\hline 22 & $23.61 \pm 3.45$ & $51.54 \pm 6.37$ & $8.86 \pm 0.08$ & $6.12 \pm 0.17$ & $1.45 \pm 0.03$ & $0.11 \pm 0.01$ & $5.48 \pm 0.11$ \\
\hline 23 & $17.88 \pm 6.76$ & $42.58 \pm 5.09$ & $7.58 \pm 0.26$ & $6.22 \pm 0.31$ & $1.22 \pm 0.06$ & $0.12 \pm 0.01$ & $4.77 \pm 0.32$ \\
\hline 24 & $17.62 \pm 4.24$ & $44.42 \pm 6.26$ & $7.50 \pm 0.16$ & $6.81 \pm 0.3$ & $1.10 \pm 0.05$ & $0.11 \pm 0.05$ & $4.74 \pm 0.44$ \\
\hline 25 & $17.62 \pm 4.88$ & $42.28 \pm 6.15$ & $7.75 \pm 0.16$ & $6.96 \pm 0.16$ & $1.11 \pm 0.07$ & $0.12 \pm 0.10$ & $4.74 \pm 0.18$ \\
\hline 26 & $14.29 \pm 6.79$ & $35.42 \pm 7.35$ & $6.89 \pm 0.11$ & $6.74 \pm 0.23$ & $1.02 \pm 0.05$ & $0.14 \pm 0.04$ & $4.27 \pm 0.01$ \\
\hline Mean & 36.34 & 32.06 & 7.95 & 7.09 & 1.12 & 0.65 & 6.72 \\
\hline $\begin{array}{l}\text { Standard } \\
\text { Deviation }\end{array}$ & 10.62 & 14.41 & 1.27 & 0.52 & 0.14 & 0.35 & 1.1 \\
\hline
\end{tabular}

Under the high glucose concentration, many RBCs adhered to each other, cells shapes changed to stomatocyte, so the roundness parameters (average value 0.65) were lower than that of low concentration. Roundness average value in table 1 reached 0.8 that implied the RBCs shapes are closed to discocyte.

Feret diameter parameters also showed that they became smaller under high glucose environment while RBCs in low glucose concentration solution remained normal cell size (figure 1b, c). Average Feret diameter in table 1 was $7.22 \mu \mathrm{m}$ that standed for a normal RBC size, at the same time this parameter was closed to the values of major axis length and minor axis length listed in the same table, which also suggested that the shape of RBCs in high glucose environment has less regularity than the normal ones. Area parameters were around $42 \mu \mathrm{m} 2$ that was more homogeneous than the values in table 2. Accordingly, a few of smaller areas values were observed in table 2, the data were thus more volatile. And this was consistent with the figurelb and $\mathrm{c}$, where many stomatocyte and adherion RBCs were found.

There were no obviously changes in average values of major axis length and minor axis length in both table, but the elongation values were different significantly. A number of elongation values were bigger up to 1.59 in figure 2 than that in figure 1. It suggested that higher glucose concentration may reduced $\mathrm{pH}$ value of solution, normal RBCs in such acidulated solution would turn to the shape of stomatocyte and acanthocyte, as shown in figure $1 \mathrm{~b}$ and $\mathrm{c}$.

Therefore, changes of glucose concentrations had obviously effect on above RBCs shape parameters. Those morphological data were in accordance with RBCs shape characteristics. Those different changes of the morphological parameters could be accurately found, analysed and calculated by MIAS.

\section{Conclusion}

The MIAS for RBCs was used to measure the morphological parameters of RBCs under normal and high glucose. Comparison of the results showed that RBCs microscopic image analysis technology was efficient on processing cells edge recognition, calculating cells sizes, roundness, area and other important morphological parameters. Those accurate quantitative results well reflected the morphologic changes of $\mathrm{RBCs}$ during certain glucose concentrations. It was known that under different glucose concentrations, the changes of the morphological parameters of RBCs reflected altered membrane protein and lipid bilayer structures. Accordingly the stability and deformation of the RBC membrane changed too. This would directly affect the performance of RBC's gases transport and other important physiological functions, which played a role in the entire cardiovascular system. Furthermore, a series of diabetic complications, for example $\mathrm{DN}$, may have close related with those changes. With the advantages of computer image processing technology, fast and accurate measuring and processing RBCs images were realized while the inconvenience of manul way was avoided. And for predicting the clinical course of DN even evaluating follow-up effect of drug treatment, the MIAS had provide a quick, intuitive and accurate approach.

\section{Acknowledgments}

The authors would like to thank professor Ping Ruan and professor Yaoxiong Huang for their guidance. This project is supported by Medical Scientific Research Foundation of Guangdong Province, P.R.China (B2014203) and Science \&Technology Planning Project of Guangdong Province, P.R.China (2016A020215162). The authors also appreciate the Laboratory in the Department of Biomedical Engineering at Jinan University for supporting this work.

\section{References}

[1] Stein P. D., Goldman J., Matta F. Yaekoub A. Y., Diabetes Mellitus and Risk of Venous Thromboembolism, American Journal of the Medical Sciences, 2009, 337 (4), 259-264

[2] Fan X., Zhang J., Theves M., Strauch C., Nemet I., Liu X., Qian J., Giblin F. J. Monnier V.M., Mechanism of lysine oxidation in human lens crystallins during aging and in diabetes, Journal of Biological Chemistry, 2009, 284 (50), 34618-34627

[3] Trachtman H., Futterweit S., Pine E., Mann J., Valderrama E., Chronic diabetic nephropathy: role of inducible nitric oxide synthase, Pediatric Nephrology, 2002, 17 (17), 20-29 
[4] Thuraisingham R. C., Nott C. A., Dodd S.M., Yaqoob M. M., Increased nitrotyrosine staining in kidneys from patients with diabetic nephropathy, Kidney international, 2000, 57 (5), 1968-1972

[5] Yong J. G., Ruan P., Feng B. H., Shi M. L. Peng L. F., Study on the relevance of changes in red blood cell morphology and function of patients with diabetic nephropathy to the development of diabetes, Journal of Jinan University (Natural Science \& Medicine Edition), 2013, 34 (6), 610-614

[6] Jiang J., Fan D. N., Chen W., Xiong Z. H., Erythrocyte counting system based on blood micrograph, Medical Information, 2009, 22 (1), 8-10

[7] Yao C. C., Huang Y. X., LI X. K., Ruan P., Effects of pH on structure and function of single living erythrocyte, Chinese Science Bulletin, 2003, 48 (13), 1342-1346
[8] Gabir M. M., Hanson R. L., Dabelea D., Imperatore G., Roumain J., Bennett P. H., Knowler W. C., The 1997 American Diabetes Association and 1999 World Health Organization criteria for hyperglycemia in the diagnosis and prediction of diabetes, Diabetes Care, 2000, 23 (8), 1108-1112

[9] Foundation N. K., K/DOQI clinical practice guidelines for chronic kidney disease: evaluation, classification, and stratification, Annals of Internal Medicine, 2003, 139 (2), $137-147$

[10] Wang J. C., Shi Z. W., The significance of urine red blood cell morphology analysis in the differential diagnosis on diabetic nephropathy and non diabetic nephropathy, Journal of Practical Medicine, 2015, 31 (1), 95-97

[11] Li H., Edge detection technology of image processing, Agriculture \& Technology, 2010, 30 (2), 163-165 